\title{
Studies of Relativistic Deuteron Reaction Cross- sections on Copper by Activation Method
}

\author{
M. Suchopár ${ }^{1 a b}$, V. Wagner ${ }^{a b}$, O. Svobodaa ${ }^{a}$ J. Vrzalováabd, P. Chudoba ${ }^{a c}$, P. Tichý ${ }^{a b}$, \\ A. Kugler ${ }^{\mathrm{a}}$ \\ ${ }^{a}$ Nuclear Physics Institute of the ASCR PRI \\ Hlavní 130, 25068 Řež, Czech Republic \\ ${ }^{b}$ Faculty of Nuclear Sciences and Physical Engineering, Czech Technical University in Prague \\ Břehová 7, 11519 Prague 1, Czech Republic \\ ${ }^{c}$ Faculty of Mathematics and Physics, Charles University in Prague \\ Ke Karlovu 3, 12116 Prague 2, Czech Republic \\ E-mail: suchopar@ujf.cas.cz, wagner@ujf.cas.cz, svoboda@ujf.cas.cz, \\ vrzalovalujf.cas.cz, chudoba@ujf.cas.cz, tichy@ujf.cas.cz, \\ kugler@ujf.cas.cz
}

J. Adam ${ }^{\text {ad }}$, L. Závorkabd, A. Baldin ${ }^{\text {de }}$, W. Furman ${ }^{d}$, M. Kadykov ${ }^{d+}$, J. Khushvaktov ${ }^{d}$, A. Solnyshkin ${ }^{d}$, V. Tsoupko-Sitnikov ${ }^{d}$, S. Tyutyunnikov ${ }^{d}$

${ }^{d}$ Joint Institute for Nuclear Research

Joliot-Curie 6, 141980 Dubna, Russia

e Institute for Advanced Studies "OMEGA"

Universitetskaya 19, 141980 Dubna, Russia

E-mail: iadamejinr.ru, zavorka@jinr.ru, Anton.Baldin@sunhe.jinr.ru,

furmanejinr.ru, kadykovejinr.ru, khushvaktovejinr.ru,

solnasunhe.jinr.ru, vtsoupkodjinr.ru, tsiesunhe.jinr.ru

\begin{abstract}
The cross-sections of relativistic deuteron reactions on natural copper were studied by means of activation method. Lack of such experimental cross-section values prevents the use of copper foils from beam integral monitoring. The copper foils were irradiated during experiments at the Joint Institute for Nuclear Research (JINR) in Dubna, Russia. The deuteron beams produced by the JINR Nuclotron accelerator had energies ranging from $1 \mathrm{GeV}$ up to $8 \mathrm{GeV}$. Residual nuclides were measured using the gamma spectrometry. The copper monitors can help us to improve the beam integral determination during further accelerator-driven system studies. Another goal of our studies is the assessment of nuclear spallation models in comparison with the experimental data.
\end{abstract}

XXII International Baldin Seminar on High Energy Physics Problems

15-20 September 2014

JINR, Dubna, Russia

${ }^{1}$ Speaker 


\section{Introduction}

The international collaboration Energy and Transmutation of Radioactive Waste (E\&T RAW) in the Joint Institute for Nuclear Research (JINR) in Dubna, Russia, performed intensive studies of simple accelerator-driven system (ADS) set-ups irradiated by proton and deuteron beams in the past years. Suitable activation detectors serve as one of possible tools for monitoring of proton and deuteron beams and for neutron field measurements in ADS studies as well.

The cross-sections of relativistic deuteron reactions on natural copper were studied in detail by means of activation method. The copper foils were irradiated during experiments with the Quinta [1] and Gamma [2] model targets in the Veksler and Baldin Laboratory of High Energies (VBLHE) in JINR. The deuteron beams with energies ranging from $1 \mathrm{GeV}$ up to 8 $\mathrm{GeV}$ were produced by the JINR Nuclotron accelerator. Residual nuclides were measured by the gamma spectrometry. Lack of such experimental cross-section values prevents the common use of copper foils from deuteron beam integral monitoring. The Nuclear Physics Institute (NPI) group commonly uses activation detectors not only for beam monitoring but also for determination of neutron field spatial distribution and proper beam integral determination preferably from multiple reactions is important for our measurements. The copper monitors will help us to improve the beam integral determination during our future ADS studies.

We use in the first place aluminium foils and ${ }^{24} \mathrm{Na}$ production reaction for the beam monitoring. Large distance from the irradiated set-up is necessary in this case due to production of ${ }^{24} \mathrm{Na}$ also by neutrons. On the other hand, the determination of the deuteron production of radionuclides on copper monitor is not influenced by $\mathrm{MeV}$ neutron reactions and such a monitor can be placed near the set-up. Thus, an improvement of our knowledge of excitation functions of various radionuclides production on copper by relativistic deuterons is necessary. These are mostly unknown unlike the proton cross-sections in the examined energy range. Therefore, we measured independent or cumulative cross-sections of different radionuclides production by deuterons on copper. Cross-sections of more than 30 isotopes were determined using the gamma spectrometry. The obtained data will enable to use copper beam monitors during ADS experiments with deuterons in the years to come. The second main goal of these studies is to provide a database for evaluation of models used for prediction of the production of different radionuclides by relativistic deuterons in various fields of application.

\section{Experimental method}

Overall sixteen irradiations were performed during five sets of experiments carried out between 2011 and 2013. Typical irradiation lasted around 12 hours with beam integral flux of the order of $10^{13}$ deuterons. As the deuteron beam integrals were determined by production of

${ }^{24} \mathrm{Na}$ in aluminium foil, our new data were in principle measured relatively to the monitoring reaction ${ }^{27} \mathrm{Al}(\mathrm{d}, \mathrm{x}){ }^{24} \mathrm{Na}$. Unfortunately, there are only three experimental cross-section values for ${ }^{27} \mathrm{Al}(\mathrm{d}, 3 \mathrm{p} 2 \mathrm{n}){ }^{24} \mathrm{Na}$ reaction in the $\mathrm{GeV}$ energy range $[3,4]$ available in the EXFOR database. Thus, a logarithmic fit between these experimental points was made to obtain the cross-section values for the given energies between the points from the EXFOR database. The aluminium and copper foils had square shape with the same size $(10 \times 10 \mathrm{~cm})$ and thicknesses of aluminium and 
copper were $0.196 \mathrm{~mm}$ and $0.128 \mathrm{~mm}$, respectively. The used copper foils had natural isotope composition (69.15\% of ${ }^{63} \mathrm{Cu}$ and $30.85 \%$ of ${ }^{65} \mathrm{Cu}$ ). Both foils were placed in the same position attached together to make sure that they were irradiated by the same beam portion. The distance from the set-up of about $3.3 \mathrm{~m}$ was sufficient to neglect the possible influence of back-scattered neutrons and other particles produced by the irradiated set-up in the direction of the beam monitors.

The activation method and gamma-ray spectrometry were used for the cross-section determination. The foils were folded up from original size to a smaller one with dimensions approximately $2.5 \times 2.5 \mathrm{~cm}$ for the spectroscopy measurement. Several high purity germanium detectors and more different geometries were used. Distances of the measured samples from the detector ranged from $4 \mathrm{~cm}$ up to $10 \mathrm{~cm}$. Every radioactive sample was measured repeatedly to detect and identify short lived as well as long lived radioisotopes. The measured gamma spectra were processed and analyzed by the DEIMOS32 spectroscopy code [8]. The yield of each isotope was calculated as a weighted average of multiple gamma lines where available. All necessary spectroscopic corrections (dead time of the detector, self-absorption correction, nonpoint like emitter correction, beam instability correction, correction for real $\gamma-\gamma$ coincidences) were taken into account. More detailed description of the used analytical procedures can be found in $[5,6,7]$.

\section{Experimental results}

Overall 38 different radioisotopes were identified by their respective gamma lines (from ${ }^{7} \mathrm{Be}$ to ${ }^{65} \mathrm{Zn}$ ) with half-life span from less than 1 hour to hundreds of days and their production cross-sections were measured using activation method. Values of the obtained partial crosssections extend over two orders of magnitude. They vary from a fraction of milibarn up to maximal values of about tens of milibarns. Most of our measured cross-sections are cumulative (for 24 radioisotopes). The rest of partial cross-sections were determined as independent (for 14 radioisotopes). Selected examples of our measured cross-sections for deuterons together with cross-sections for proton reactions from the EXFOR database can be found in Figures 1-6.

(a)

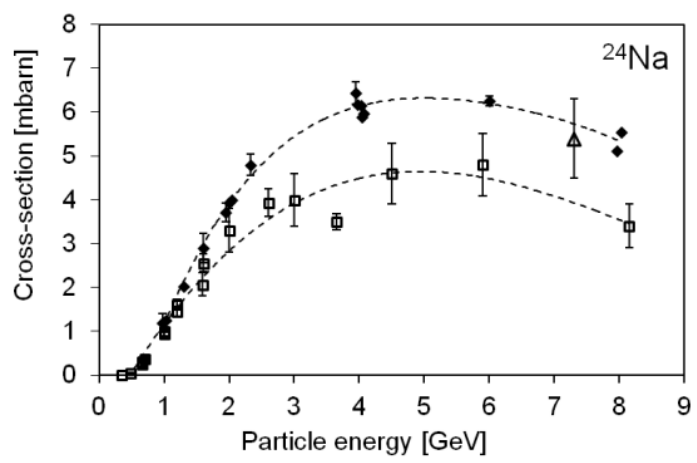

(b)

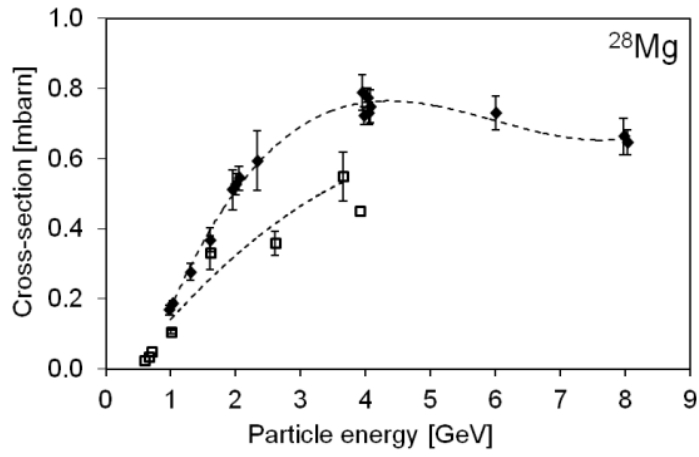

Fig.1: (a) Cross-sections of ${ }^{n a t} \mathrm{Cu}(\mathrm{d}, \mathrm{x})^{24} \mathrm{Na}$ reaction (our data - rhombs, EXFOR data - triangle) and ${ }^{\text {nat }} \mathrm{Cu}(\mathrm{p}, \mathrm{x})^{24} \mathrm{Na}$ reaction (EXFOR data - empty squares), (b) Cross-sections of ${ }^{\text {nat }} \mathrm{Cu}(\mathrm{d}, \mathrm{x})^{28} \mathrm{Mg}$ reaction (our data - rhombs) and ${ }^{\text {nat }} \mathrm{Cu}(\mathrm{p}, \mathrm{x})^{28} \mathrm{Mg}$ reaction (EXFOR data - empty squares) 
(a)

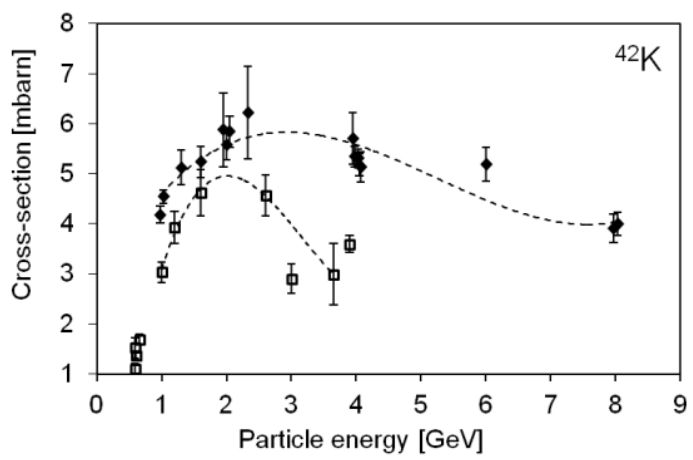

(b)

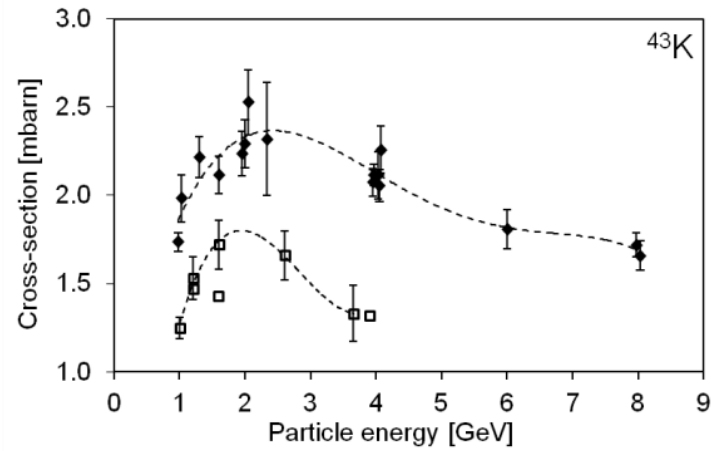

Fig.2: (a) Cross-sections of ${ }^{\text {nat }} \mathrm{Cu}(\mathrm{d}, \mathrm{x})^{42} \mathrm{~K}$ reaction (our data - rhombs) and ${ }^{\text {nat }} \mathrm{Cu}(\mathrm{p}, \mathrm{x})^{42} \mathrm{~K}$ reaction (EXFOR data - empty squares), (b) Cross-sections of ${ }^{\text {nat }} \mathrm{Cu}(\mathrm{d}, \mathrm{x}){ }^{43} \mathrm{~K}$ reaction (our data - rhombs) and ${ }^{\text {nat }} \mathrm{Cu}(\mathrm{p}, \mathrm{x}){ }^{43} \mathrm{~K}$ reaction (EXFOR data - empty squares)

(a)

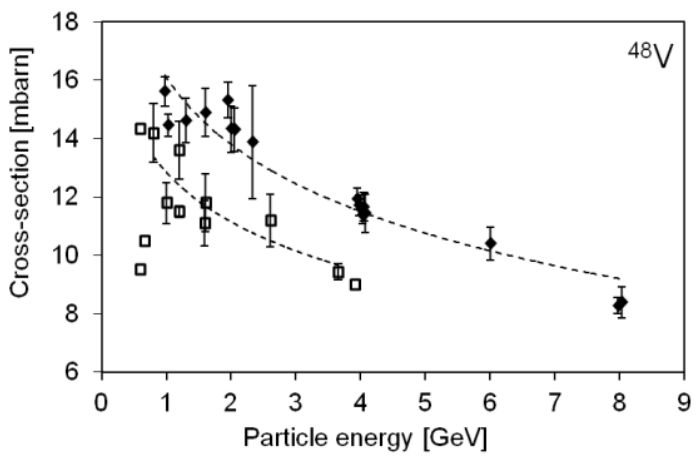

(b)

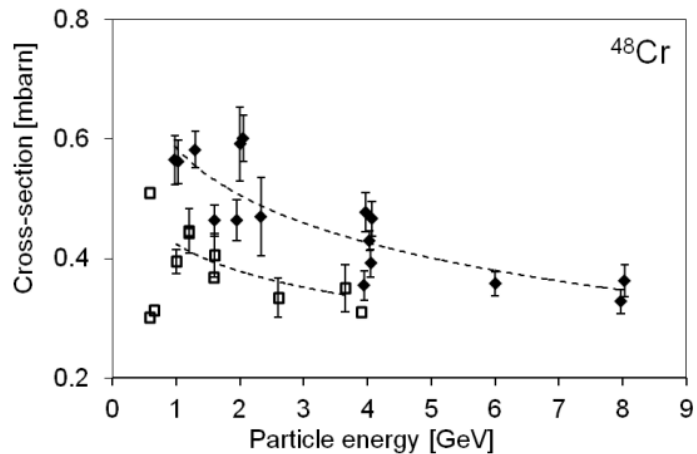

Fig.3: (a) Cross-sections of ${ }^{\text {nat }} \mathrm{Cu}(\mathrm{d}, \mathrm{x})^{48} \mathrm{~V}$ reaction (our data - rhombs) and ${ }^{\text {nat }} \mathrm{Cu}(\mathrm{p}, \mathrm{x})^{48} \mathrm{~V}$ reaction (EXFOR data - empty squares), (b) Cross-sections of nat $\mathrm{Cu}(\mathrm{d}, \mathrm{x}){ }^{48} \mathrm{Cr}$ reaction (our data - rhombs) and ${ }^{\text {nat }} \mathrm{Cu}(\mathrm{p}, \mathrm{x}){ }^{48} \mathrm{Cr}$ reaction (EXFOR data - empty squares)

(a)

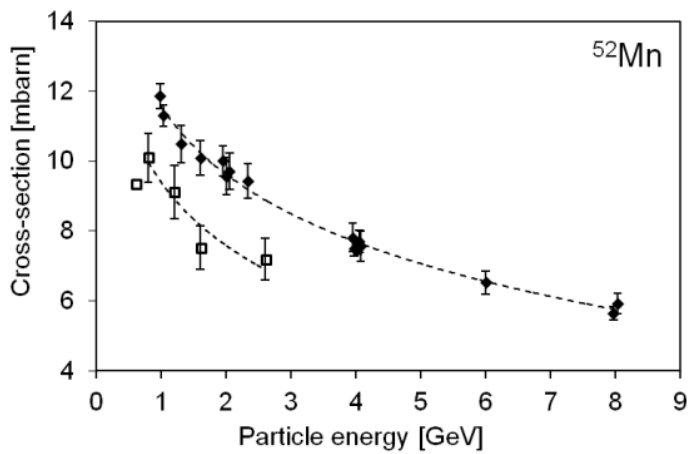

(b)

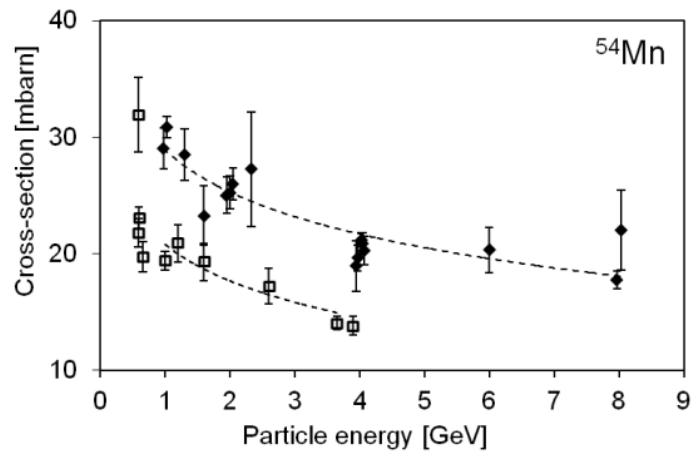

Fig.4: (a) Cross-sections of ${ }^{\text {nat }} \mathrm{Cu}(\mathrm{d}, \mathrm{x})^{52} \mathrm{Mn}$ reaction (our data - rhombs) and ${ }^{\text {nat }} \mathrm{Cu}(\mathrm{p}, \mathrm{x})^{52} \mathrm{Mn}$ reaction (EXFOR data - empty squares), (b) Cross-sections of nat $\mathrm{Cu}(\mathrm{d}, \mathrm{x})^{54} \mathrm{Mn}$ reaction (our data - rhombs) and ${ }^{\text {nat }} \mathrm{Cu}(\mathrm{p}, \mathrm{x}){ }^{54} \mathrm{Mn}$ reaction (EXFOR data - empty squares) 
(a)

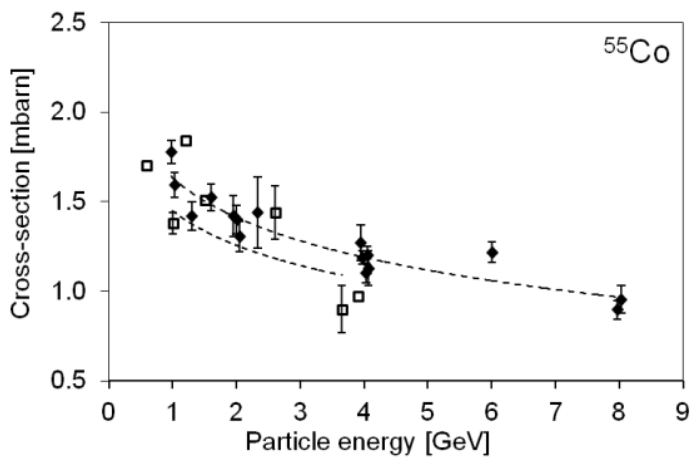

(b)

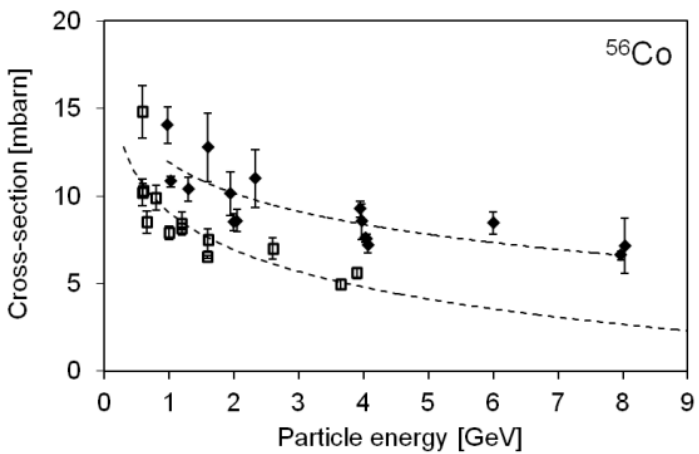

Fig.5: (a) Cross-sections of ${ }^{\text {nat }} \mathrm{Cu}(\mathrm{d}, \mathrm{x}){ }^{55} \mathrm{Co}$ reaction (our data - rhombs) and ${ }^{\text {nat }} \mathrm{Cu}(\mathrm{p}, \mathrm{x}){ }^{55} \mathrm{Co}$ reaction (EXFOR data - empty squares), (b) Cross-sections of ${ }^{\text {nat }} \mathrm{Cu}(\mathrm{d}, \mathrm{x}){ }^{56} \mathrm{Co}$ reaction (our data rhombs) and ${ }^{\text {nat }} \mathrm{Cu}\left(\mathrm{p}, \mathrm{x}{ }^{56} \mathrm{Co}\right.$ reaction (EXFOR data - empty squares)

(a)

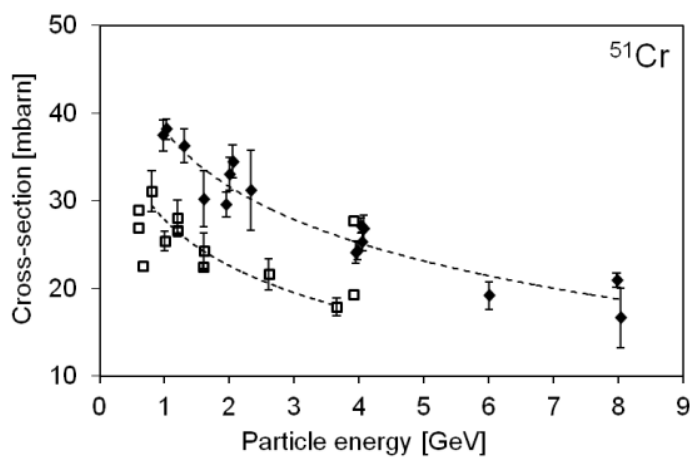

(b)

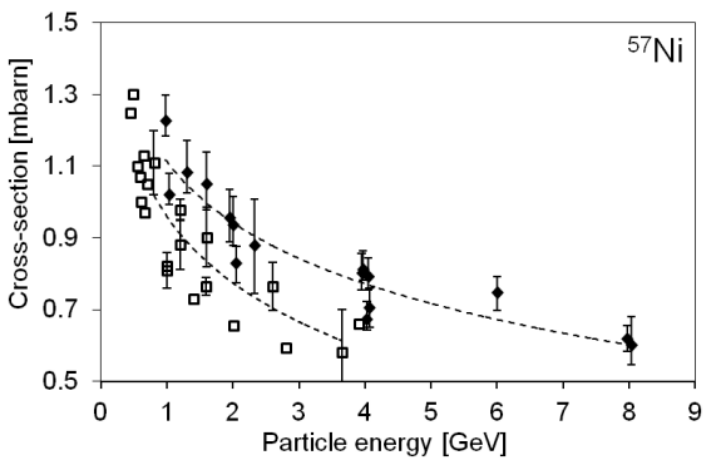

Fig.6: (a) Cross-sections of ${ }^{n a t} \mathrm{Cu}(\mathrm{d}, \mathrm{x}){ }^{51} \mathrm{Cr}$ reaction (our data - rhombs) and ${ }^{\text {nat }} \mathrm{Cu}(\mathrm{p}, \mathrm{x}){ }^{51} \mathrm{Cr}$ reaction (EXFOR data - empty squares), (b) Cross-sections of ${ }^{\text {nat }} \mathrm{Cu}(\mathrm{d}, \mathrm{x})^{57} \mathrm{Ni}$ reaction (our data rhombs) and ${ }^{\text {nat }} \mathrm{Cu}(\mathrm{p}, \mathrm{x})^{57} \mathrm{Ni}$ reaction (EXFOR data - empty squares)

\section{Simulations}

Deuteron cross-sections simulations were made both in the deterministic code TALYS version 1.6 [10] and the Monte Carlo code MCNPX version 2.7.0 [9] with two intra-nuclear cascade-evaporation models INCL-ABLA and LAQGSM included in the code. The optical potential model used in the TALYS code is obviously not suitable for the description of spallation reactions initiated by deuterons in the energy range 500-1000 MeV. The models used in the MCNPX code are more suitable for the description of the excitation function of deuterons impacting on natural copper in the energy range 1-8 GeV. The experimental data are better fitted with the LAQGSM model than the INCL-ABLA model in most cases. The simulated excitation functions are generally similar in shape but can differ significantly from the experimental data in absolute value in certain cases. Selected examples of our measured cross-sections for deuterons together with excitation functions for deuteron reactions simulated in the TALYS and MCNPX codes are displayed in Figures 7-12. 
(a)

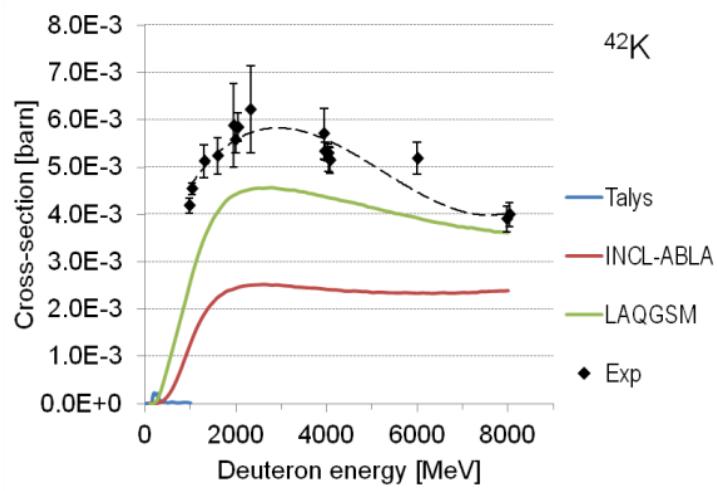

(b)

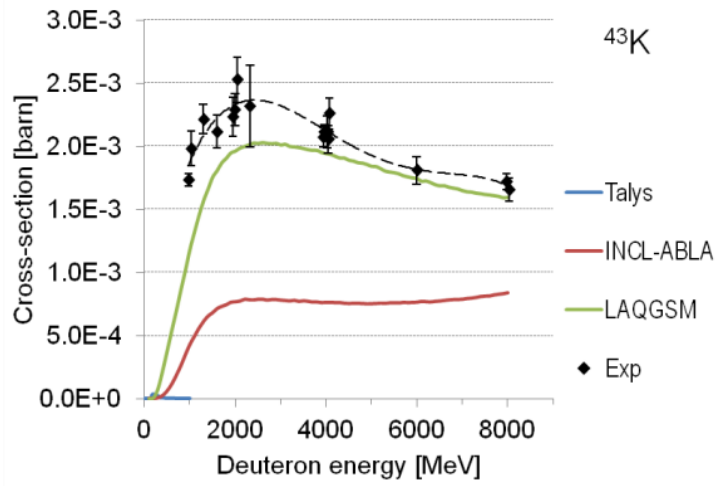

Fig.7: (a) Cross-sections of ${ }^{\text {nat }} \mathrm{Cu}(\mathrm{d}, \mathrm{x})^{42} \mathrm{~K}$ reaction (our data - rhombs) and simulated excitation functions (TALYS + MCNPX), (b) Cross-sections of ${ }^{\text {nat }} \mathrm{Cu}(\mathrm{d}, \mathrm{x}){ }^{43} \mathrm{~K}$ reaction (our data - rhombs) and simulated excitation functions (TALYS + MCNPX)

(a)

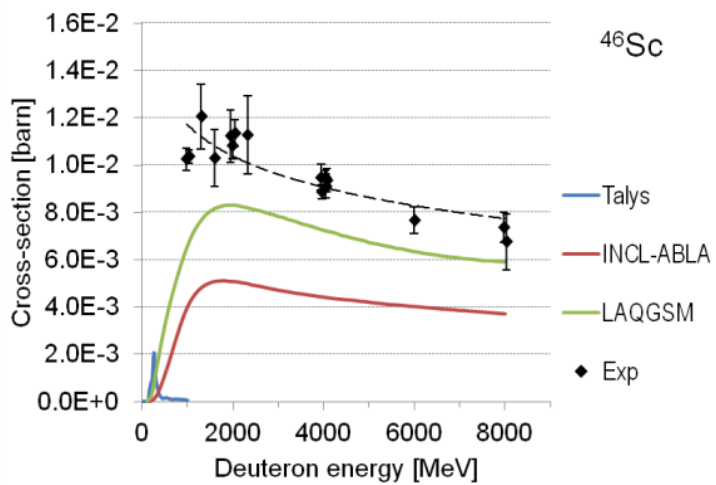

(b)

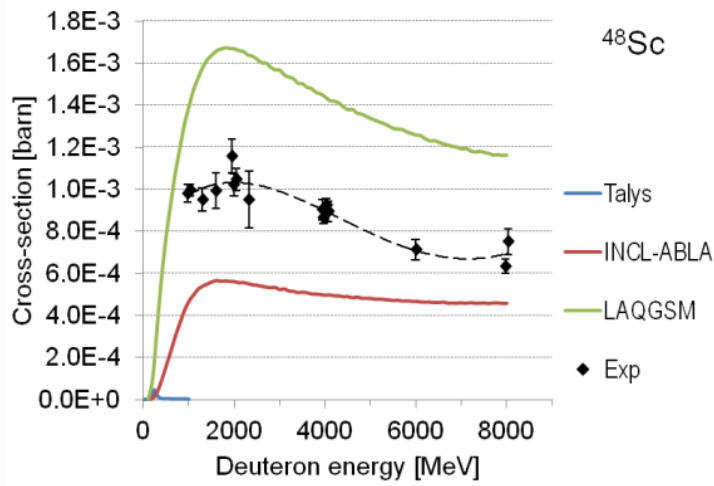

Fig.8: (a) Cross-sections of ${ }^{\text {nat }} \mathrm{Cu}(\mathrm{d}, \mathrm{x})^{46} \mathrm{Sc}$ reaction (our data - rhombs) and simulated excitation functions (TALYS + MCNPX), (b) Cross-sections of ${ }^{\text {nat }} \mathrm{Cu}(\mathrm{d}, \mathrm{x})^{48} \mathrm{Sc}$ reaction (our data - rhombs) and simulated excitation functions (TALYS + MCNPX)

(a)

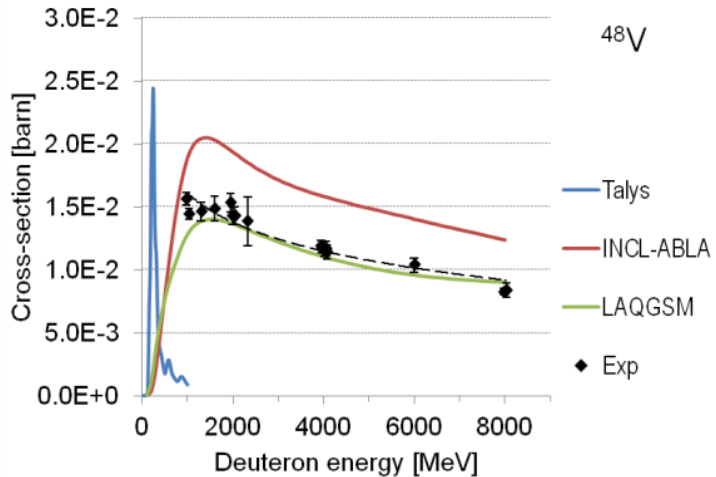

(b)

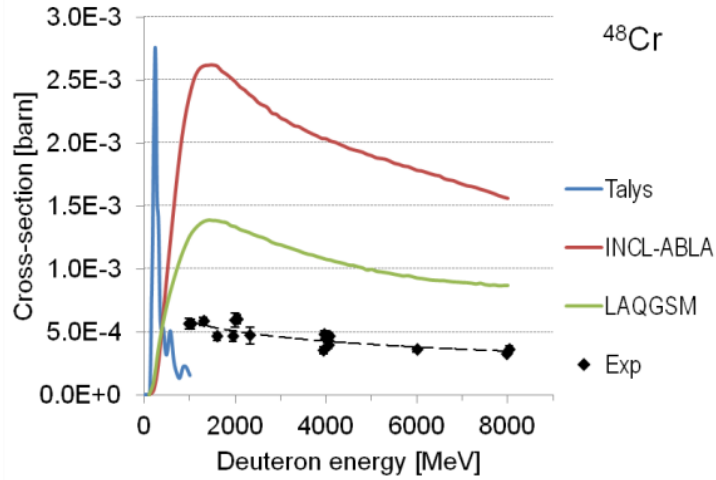

Fig.9: (a) Cross-sections of ${ }^{\text {nat }} \mathrm{Cu}(\mathrm{d}, \mathrm{x})^{48} \mathrm{~V}$ reaction (our data - rhombs) and simulated excitation functions (TALYS + MCNPX), (b) Cross-sections of ${ }^{n a t} \mathrm{Cu}(\mathrm{d}, \mathrm{x}){ }^{48} \mathrm{Cr}$ reaction (our data - rhombs) and simulated excitation functions (TALYS + MCNPX) 
(a)

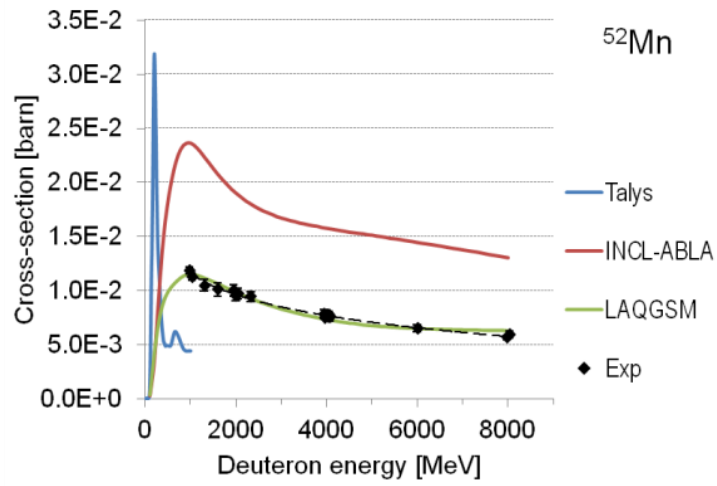

(b)

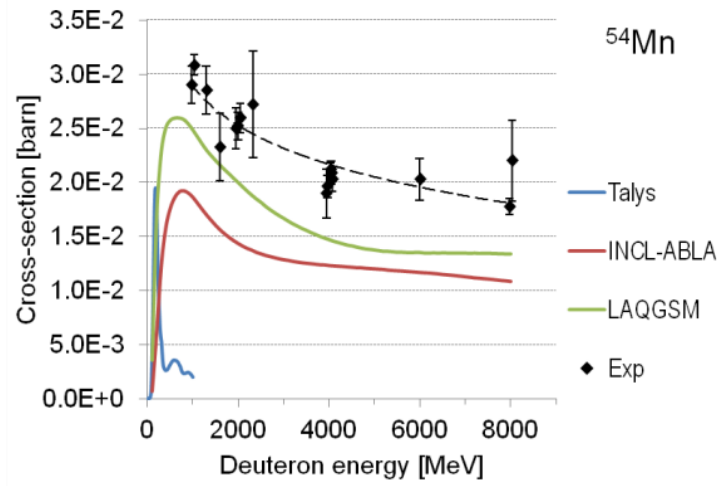

Fig.10: (a) Cross-sections of ${ }^{\text {nat }} \mathrm{Cu}(\mathrm{d}, \mathrm{x})^{52} \mathrm{Mn}$ reaction (our data - rhombs) and simulated excitation functions (TALYS + MCNPX), (b) Cross-sections of ${ }^{n a} \mathrm{Cu}(\mathrm{d}, \mathrm{x}){ }^{54} \mathrm{Mn}$ reaction (our data - rhombs) and simulated excitation functions (TALYS + MCNPX)

(a)

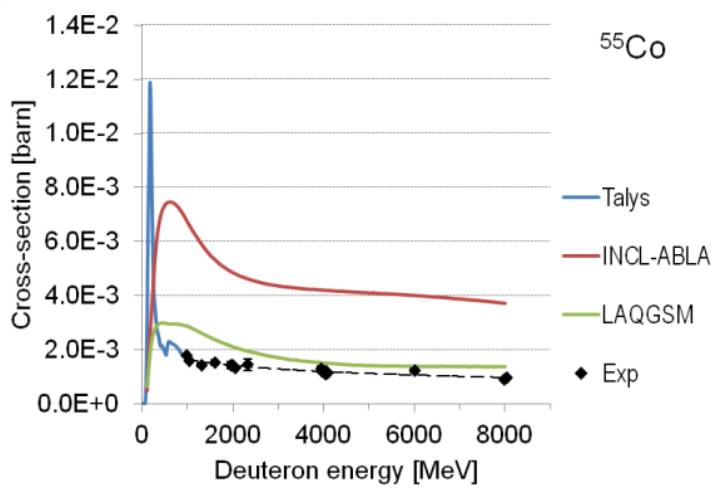

(b)

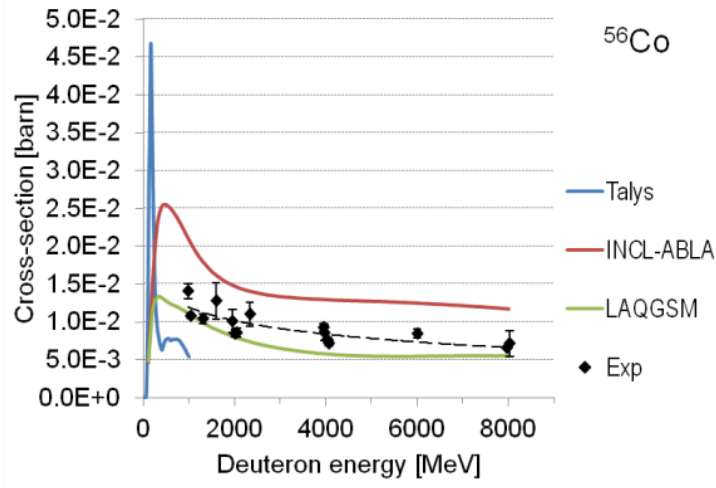

Fig.11: (a) Cross-sections of ${ }^{\text {nat }} \mathrm{Cu}(\mathrm{d}, \mathrm{x}){ }^{55} \mathrm{Co}$ reaction (our data - rhombs) and simulated excitation functions (TALYS + MCNPX), (b) Cross-sections of ${ }^{\text {nat }} \mathrm{Cu}(\mathrm{d}, \mathrm{x}){ }^{56} \mathrm{Co}$ reaction (our data - rhombs) and simulated excitation functions (TALYS + MCNPX)

(a)

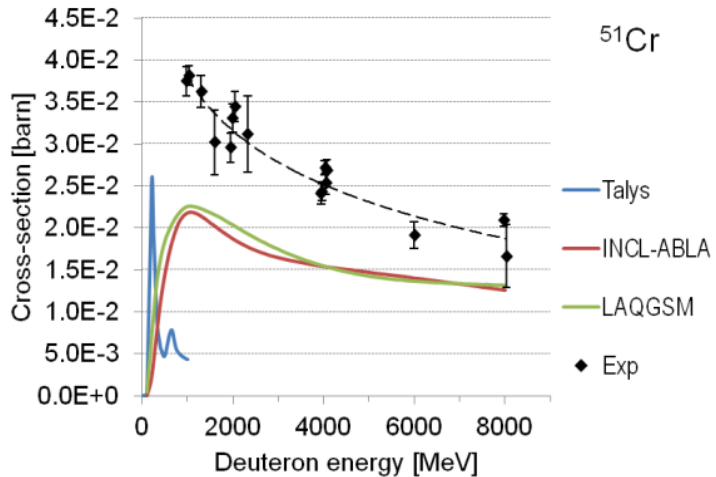

(b)

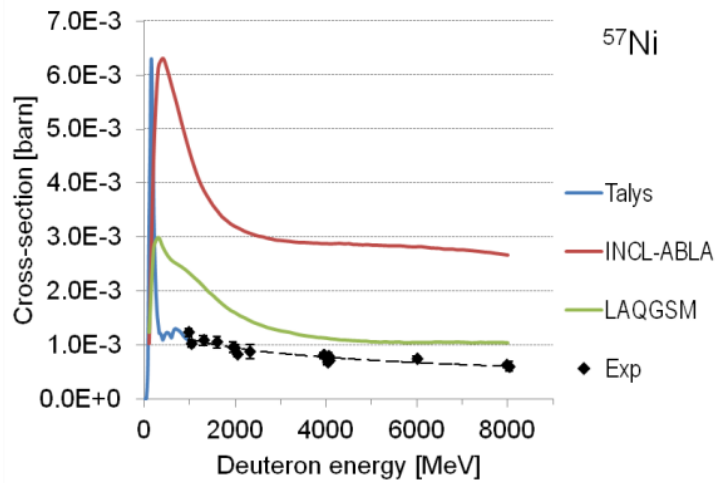

Fig.12: (a) Cross-sections of nat $\mathrm{Cu}(\mathrm{d}, \mathrm{x})^{51} \mathrm{Cr}$ reaction (our data - rhombs) and simulated excitation functions (TALYS + MCNPX), (b) Cross-sections of nat $\mathrm{Cu}(\mathrm{d}, \mathrm{x})^{57} \mathrm{Ni}$ reaction (our data - rhombs) and simulated excitation functions (TALYS + MCNPX) 


\section{Conclusion}

Within the Energy and Transmutation framework, the excitation functions of relativistic deuteron reactions on natural copper were studied by activation method in the energy range from 1 to $8 \mathrm{GeV}$. The partial cross-sections of nearly forty different radionuclides produced in the activation foil were determined. The investigated residual nuclei cover gamma emitting radionuclides with half-lives extending between a couple of hours and several years.

The excitation functions of relativistic deuteron reactions on copper were compared to those for relativistic protons extracted from the EXFOR database and they show very similar trends. The absolute values of partial cross-sections for deuteron reactions are in general higher by more than $30 \%$ than those for proton reactions.

The new set of experimental partial cross-sections measured by our group was used to test nuclear reaction models of high-energy reactions such as spallation and fragmentation. Mainly different models used by the MCNPX code were tested. The obtained cross-sections will be also useful for monitoring of deuteron beam by copper foils in our future experiments.

\section{References}

[1] W. Furman et al, Recent results of the study of ADS with $500 \mathrm{~kg}$ natural uranium target assembly QUINTA irradiated by deuterons with energies from 1 to $8 \mathrm{GeV}$ at JINR NUCLOTRON, Baldin ISHEPP XXI 086, in Proceedings of Science (2012)

[http://pos.sissa.it/archive/conferences/173/086/Baldin\%20ISHEPP\%20XXI_086.pdf]

[2] J. Adam, C. Bhatia, V. Kumar, K. Katovský, M. Majerle et al, A study of reaction rates of $(n, f)$, $(n, g)$ and $(n, 2 n)$ reactions in $U$-nat and Th-232 by the neutron fluence produced in the graphite setup (GAMMA-3) irradiated by $2.33 \mathrm{GeV}$ deuteron beam, European Physical Journal A 47 (2011) 85

[3] J. Banaigs et al, Determination De L'Intensite D'Un Faisceau De Deutons Extrait D'Un Synchrotron Et Mesure Des Sections Efficaces Des Reactions C-12(D,P2N)C-11 Et Al27(D,3P2N)Na-24 a $2.33 \mathrm{GeV}$, Nuclear Instruments and Methods in Physics Research 95 (1971) $307-311$

[4] P. Kozma and V. V. Yanovski, Application of $\mathrm{BaF}_{2}$ scintillator to off-line gamma ray spectroscopy, Czech Journal of Physics 40 (1990) 393

[5] V. Wagner et al, Studies of deuteron and neutron cross-sections important for ADS research, Baldin ISHEPP XXI 090, in Proceedings of Science (2012)

[http://pos.sissa.it/archive/conferences/173/090/Baldin\%20ISHEPP\%20XXI_090.pdf]

[6] V. Wagner et al, Cross-section studies of important neutron and relativistic deuteron reactions, XX International School on Nuclear Physics, Neutron Physics and Applications, in Journal of Physics: Conference Series 533 (2014) [http://iopscience.iop.org/1742-6596/533/1/012052]

[7] V. Wagner et al, Cross-section studies of relativistic deuteron reactions obtained by activation method, in ERINDA Workshop Proceedings (2013) p. 103

[http://indico.cern.ch/event/251800/material/1/0.pdf]

[8] J. Frána, Program DEIMOS32 for gamma-ray spectra evaluation, Journal of Radioanalytical and Nuclear Chemistry 257 (2003) 583-587

[9] D. B. Pelowitz, MCNPX User's Manual Version 2.6.0, LA-CP-07-1473, LANL (2008)

[10] A. J. Koning, D. Rochman, Modern Nuclear Data Evaluation with the TALYS Code System, Nuclear Data Sheets, Vol. 113 (2012) 2841-2934 